\title{
Diabetes na gestação
}

\author{
Diabetes in pregnancy
}

\section{Diabetes en la gestación}

\author{
Raquel C. Abi-Abib, Carolina A. Cabizuca, João Regis I. Carneiro," Fernanda O. Braga, \\ Roberta A. Cobas, Marília B. Gomes, Guilherme R. de Jesús, Fátima R. D. Miranda
}

\begin{abstract}
Resumo
Devido ao aumento da idade materna e à epidemia de obesidade, a prevalência de gestantes com diabetes tipo 2 ou que desenvolvem diabetes mellitus gestacional (DMG) tem sido cada vez maior. A associação do diabetes com aumento do risco de complicações materno-fetais é conhecida há muitos anos, mas foi apenas em 2008 que a forte e contínua relação entre níveis glicêmicos acima do normal e risco de complicações fetais foi confirmada por meio de um grande ensaio clínico prospectivo. Atualmente, a investigação quanto à presença de diabetes na gestação é recomendada para todas as gestantes, pela realização de glicemia de jejum na primeira consulta do pré-natal e de TOTG (Teste Oral de Tolerância à Glicose) entre a $24^{\mathrm{a}}$ e a $28^{\mathrm{a}}$ semanas. O tratamento realizado por equipe multidisciplinar e com objetivo de manter o controle glicêmico o mais próximo possível do normal possibilita a redução das taxas de morbimortalidade materno-fetais. O objetivo desta revisão é discorrer sobre epidemiologia, fisiopatologia, diagnóstico, complicações, acompanhamento e tratamento de gestantes portadoras de diabetes tipo 1 ou 2 e das portadoras de DMG, ressaltando-se as semelhanças e peculiaridades de cada caso, com base nos principais estudos e consensos mais recentes.
\end{abstract}

Descritores: Diabetes mellitus; Diabetes gestacional; Gestação.

\begin{abstract}
Due to maternal aging and the obesity epidemic, the prevalence of type 2 diabetes mellitus among pregnant women and gestational diabetes mellitus is increasing. The association of diabetes and increasing risk of maternal and fetal complications has been known for several years, but only in 2008 the strong and continuous relationship between high glucose levels and the risk of fetal complications was confirmed in a large prospective clinical trial. Currently, diabetes screening is warranted in every pregnant woman through the measurement of fasting plasma glucose in the first pre-natal evaluation and OGTT (Oral Glucose Tolerance Test) between the $24^{\text {th }}$ and $28^{\text {th }}$ gestational weeks. Treatment is carried out by a multidisciplinary team, aiming at achieving near-normal glucose levels, leading to the reduction of maternal and fetal morbidity. The purpose of this review article is to discuss the epidemiology, physiopathology, diagnosis, complications, treatment and follow-up of pregnant women with pregestational diabetes and gestational diabetes, pointing out the similarities and peculiarities of each condition based on the most important studies and the most recent consensus.
\end{abstract}

Keywords: Diabetes mellitus; Diabetes, Gestational; Pregnancy.

\section{Resumen}

Debido al aumento de la edad materna y a la epidemia de obesidad, la prevalencia de gestantes con diabetes tipo 2 o que desarrollan diabetes mellitus gestacional (DMG) ha sido cada vez mayor. La asociación de la diabetes con aumento del riesgo de complicaciones materno-fetales es conocida hace muchos anos, pero fue apenas el 2008 que la fuerte y continua relación entre niveles glicémicos por encima de lo normal y riesgo de complicaciones fetales fue confirmada por medio de un gran ensayo clínico prospectivo. Actualmente, la investigación en cuanto a la presencia de diabetes en la gestación es recomendada para todas las gestantes, mediante la realización de glicemia de ayuno en la primera consulta del prenatal y el Test de tolerancia oral a

Revista HUPE, Rio de Janeiro, 2014;13(3):40-47

Recebido: 24/04/2014 | Revisado: 02/07/2014 | Aprovado: 28/07/2014

doi: 10.12957/rhupe.2014.12136
"Endereço para correspondência: Serviço de Diabetes, Hospital Universitário Pedro Ernesto. Boulevard 28 de Setembro, 77, 3ㅇandar. Rio de Janeiro, RJ. CEP: 20551-030. E-mail: endoregis@uol.com.br 
la glucosa (TTOG) entre la $24^{\mathrm{a}}$ y la $28^{\mathrm{a}}$ semanas. El tratamiento realizado por equipo multidisciplinario, con el objetivo de mantener control glicémico lo más próximo posible de lo normal posibilita la reducción de las tasas de morbimortalidad materno-fetales. El objetivo de esta revisión es discurrir sobre la epidemiología, fisiopatología, diagnóstico, complicaciones, acompañamiento y tratamiento de gestantes portadoras de diabetes tipo 1 o 2 y de las portadoras de DMG, resaltándose las semejanzas y peculiaridades de cada caso, con base en los principales estudios y consensos más recientes.

Palabras clave: Diabetes mellitus, Diabetes gestacional; Gestación.

\section{Introdução}

A gestação complicada pelo diabetes, seja ele pré-gestacional ou gestacional, tem um risco elevado de morbimortalidade materna e fetal. $\mathrm{O}$ planejamento da gestação, rastreamento para o diabetes mellitus gestacional (DMG) e o acompanhamento com uma equipe multidisciplinar podem minimizar os riscos. Além disso, gestantes com diabetes pré-gestacional podem apresentar agravamento das complicações crônicas microvasculares e no controle glicêmico, já que a gravidez é um estado diabetogênico. Com o aumento da epidemia de obesidade, cada vez mais mulheres em idade fértil apresentam risco de desenvolvimento de diabetes tipo 2 e DMG. A prevalência deste último varia de 3 a $14 \%$ dependendo da população estudada e do método utilizado para sua detecção. Este artigo tem como objetivo rever os principais tópicos relacionados à gestação complicada pelo diabetes.

\section{Fisiopatologia}

Durante a gestação, o metabolismo materno se adapta para suprir as necessidades de nutrientes da unidade feto-placentária. No primeiro trimestre, a glicose passa para o feto por difusão facilitada e os aminoácidos são transportados ativamente para a circulação placentária. A diminuição nos níveis de glicose e a perda de substrato para a gliconeogênese (principalmente do aminoácido alanina) são fatores importantes para a hipoglicemia materna no início da gestação. Por volta da $18^{\mathrm{a}}$ semana, tem início a resistência à ação da insulina que progride no terceiro trimestre a níveis semelhantes àqueles observados no diabetes mellitus tipo 2 (DM2). A sensibilidade periférica à insulina no terceiro trimestre cai em $50 \%$, e a produção hepática de glicose é 30\% maior do que no início da gestação. ${ }^{1}$ A resistência insulínica resulta da combinação entre o aumento da adiposidade materna e da produção placentária de hormônios diabetogênicos, incluindo hormônio do crescimento, cortisol, hormônio lactogênico placentário e progesterona.

\section{Diagnóstico de DMG}

Até março de 2010, com a publicação pela IADPSG (International Association of Diabetes and Pregnancy Study Groups) dos novos critérios diagnósticos de DMG, existiam várias formas de rastreamento, ainda sem um consenso universal, sobre qual o melhor teste a ser utilizado. ${ }^{2}$

Os critérios do IADPSG foram fundamentados no maior estudo multicêntrico já realizado em gestantes. Publicado em 2008, o objetivo desse estudo era avaliar a associação entre os diversos graus de hiperglicemia materna e o aumento dos riscos de complicações materno-fetais. ${ }^{3}$ No estudo HAPO (Hyperglycemia and Adverse Pregnancy Outcomes), 25.505 gestantes sem diagnóstico de diabetes foram submetidas ao TOTG com 75 g de glicose anidra entre a $24^{\mathrm{a}}$ e $32^{\mathrm{a}}$ semana de gestação. Os pesquisadores concluíram que existe uma associação direta entre os níveis de glicemia maternos abaixo daqueles que eram considerados diagnósticos de diabetes e o aumento do peso fetal e dos níveis de peptídeo $\mathrm{C}$ no cordão umbilical.

Tendo em vista os resultados do HAPO, um novo consenso foi publicado em 2010 com o objetivo de uniformizar os critérios para diagnóstico de DMG. ${ }^{2}$ A recomendação atual é que todas as gestantes realizem uma glicemia de jejum na primeira consulta pré-natal. Se o resultado for menor que 92 mg/dL, um TOTG com 75 g deverá ser realizado entre a $24^{\mathrm{a}}$ e $28^{\mathrm{a}}$ semana de gestação. Caso a glicemia esteja acima de $126 \mathrm{mg} / \mathrm{dL}$, o diagnóstico será de diabetes pré-gestacional, e se estiver acima de 92 e menor que 126 mg/dL, o diagnóstico de DMG está estabelecido. Os resultados devem ser confirmados com uma segunda medida da glicemia. Caso seja necessário realizar o TOTG, a dosagem da glicemia deverá ser feita em jejum, 1 e 2 horas após a sobrecarga de glicose. 
Tabela 1. Critérios para o diagnóstico de DMG da International Association of Diabetes and Pregnancy Study Groups -2010.

\begin{tabular}{c|c}
\hline Tempo & Valor máximo da normalidade \\
\hline Basal & $92 \mathrm{mg} / \mathrm{dL}$ \\
\hline 1 hora & $180 \mathrm{mg} / \mathrm{dL}$ \\
\hline 2 horas & $153 \mathrm{mg} / \mathrm{dL}$ \\
\hline
\end{tabular}

Diagnóstico de DMG com 1 ou mais valores $\geq$ aos acima²

Apenas um valor acima do limite estabelecido é suficiente para o diagnóstico de DMG (Tabela 1).

\section{Complicações materno-fetais}

Mulheres com diabetes pré-gestacional diferem daquelas com DMG porque a hiperglicemia poderá estar presente no momento da organogênese fetal, aumentando substancialmente o risco de malformações congênitas. Estas são observadas em 6 a 12\% dos filhos de mães diabéticas. As anomalias congênitas mais comuns são as cardíacas, esqueléticas e neurológicas, sendo a síndrome de regressão caudal de 250 a 600 vezes mais frequente em filhos de mães diabéticas. ${ }^{4}$

A macrossomia fetal, definida quando o peso do recém-nato é maior que 4 kg na gestação a termo ou maior que o percentil 90 para a idade gestacional, é a manifestação mais característica. É observada em até 30\% dos casos e pode predispor a traumas obstétricos e distocia de ombro. ${ }^{5}$ A hiperinsulinemia fetal, que resulta do diabetes materno, é um fator importante no crescimento fetal excessivo. Outros fatores maternos além da hiperglicemia, como obesidade e concentração sérica de aminoácidos e lipídeos, também influenciam o peso fetal. ${ }^{6}$ Filhos hiperinsulinêmicos de mães diabéticas possuem um risco aumentado de hipoglicemia após o parto. Esta complicação se torna menos comum em filhos de gestantes que permaneceram euglicêmicas durante o parto e na maior parte da gestação. ${ }^{6,7}$

Outras complicações neonatais relacionadas ao DMG são hiperbilirrubinemia, a hipocalcemia, o retardo de crescimento intrauterino, a policitemia e a síndrome de angústia respiratória. ${ }^{7}$ Estudos demonstram que indivíduos expostos ao diabetes materno (tipo 1, 2 ou DMG) intraútero têm aumento do risco de obesidade e intolerância à glicose na infância e no início da vida adulta. ${ }^{8}$ Além dos resultados adversos fetais, complicações mater- nas também são relevantes, especialmente na presença de retinopatia e nefropatia, sobretudo naquelas que já apresentavam tais complicações previamente.

Mulheres com DMG têm um risco aumentado de desenvolverem pré-eclâmpsia (de 10 a $30 \%$ ) quando comparadas àquelas com tolerância normal à glicose (de 5 a $7 \%$ de risco). ${ }^{32}$ Além disso, DMG aumenta substancialmente a chance de desenvolver diabetes após o parto, de acordo com alguns estudos mostrando que este risco é de aproximadamente $40 \%$ após um período de seguimento de 10 anos. $^{10}$

\section{Aconselhamento pré-natal nas diabéticas prévias}

A paciente diabética deve ser orientada, desde a puberdade, sobre os riscos de uma gravidez não planejada e a importância de um bom controle glicêmico anterior à gestação. Idealmente, os níveis glicêmicos e a hemoglobina glicada devem ficar o mais próximo da normalidade. Ela deve ser avaliada também quanto à presença de complicações crônicas como nefropatia, retinopatia, neuropatia e cardiopatia. A pressão arterial deve estar controlada, com valores < 130/80 mmHg, e os inibidores da enzima conversora de angiotensina e bloqueadores do receptor da angiotensina devem ser descontinuados antes da concepção. Nas diabéticas tipo 1, a função tireoidiana deve ser avaliada com objetivo de manter o TSH entre 1,3 e $2,5 \mu \mathrm{U} / \mathrm{L}$. No quadro 1 estão resumidas as contraindicações potenciais para gravidez em diabéticas."

\section{Objetivos do tratamento}

Sabe-se que há uma associação contínua entre os valores glicêmicos na gestação e a taxa de complicações materno-fetais. No entanto, o limiar 


\section{Quadro 1.}

\begin{tabular}{|l|}
\hline $\begin{array}{l}\text { Contraindicações potenciais para gravidez em } \\
\text { diabéticas }\end{array}$ \\
\hline Doença cardíaca isquêmica \\
Hipertensão arterial $>140 / 90 \mathrm{mmHg}$, apesar do tratamento \\
Gastroparesia \\
Retinopatia proliferativa não tratada \\
$\begin{array}{l}\text { Nefropatia diabética grave: clearance de creatinina }<50 \\
\mathrm{ml} / \mathrm{min} \text { e/ou creatinina sérica }>2 \mathrm{mg} / \mathrm{dL} \text { e/ou proteinúria }> \\
2 \mathrm{~g} / 24 \mathrm{~h}\end{array}$ \\
\hline
\end{tabular}

de glicemia a partir do qual o risco de complicações aumenta de forma significativa não é claro. ${ }^{3}$ Estudos de revisão sugerem que o ponto de corte para glicemia de jejum de $90 \mathrm{mg} / \mathrm{dL}$ seria o mais relacionado à redução do risco de macrossomia; entretanto, metas de glicemia muito rigorosas aumentam o risco de hipoglicemia e podem não ser seguras para a maior parte das gestantes. ${ }^{12}$

Com o objetivo de minimizar o risco de complicações materno-fetais, o tratamento do diabetes na gestação tem como meta valores de glicemia pré-prandiais inferiores a $95 \mathrm{mg} / \mathrm{dL}, 1$ hora após o início da refeição inferior a $140 \mathrm{mg} / \mathrm{dL}$, e 2 horas pós-prandial abaixo de 120 mg/dL."113

\section{Tratamento}

\section{Dieta}

A orientação nutricional é parte fundamental do tratamento de toda gestante portadora de diabetes. O cálculo calórico diário deve ser individualizado de acordo com IMC, nível de atividade física e crescimento fetal. Em geral, calcula-se a ingesta calórica diária de 30 a 35 kcal/kg/dia para gestantes com peso normal e $25 \mathrm{kcal} / \mathrm{kg} /$ dia para aquelas com sobrepeso ou obesidade. Dietas com menos de $1500 \mathrm{kcal} /$ dia associam-se ao risco de cetoacidose e precisam ser evitadas. ${ }^{14}$ As recomendações de ganho de peso durante a gestação e a cada semana do $2^{\circ}$ e do $3^{\circ}$ trimestres estão descritas na tabela $2 . .^{15}$

O conteúdo calórico total deve ser composto por 40 a $45 \%$ de carboidratos, 15 a 20\% de proteínas e 30 a $40 \%$ de gorduras e dividido em cerca de seis refeições leves ao longo do dia. Deve-se optar por carboidratos complexos e ricos em fibras. Os adoçantes não caló- ricos aspartame, sucralose e acessulfame podem ser utilizados na gestação, de forma moderada. ${ }^{13}$

\section{Exercícios físicos}

Exercícios físicos promovem redução da resistência insulínica, levando à melhora do controle glicêmico, à redução do ganho de peso e à diminuição da adiposidade fetal, além de ampliar a tolerância ao parto. Na ausência de contraindicações, deve-se recomendar a prática de atividade física por pelo menos 30 minutos, na maior parte dos dias da semana, evitando-se exercícios com risco de queda ou trauma abdominal. . $^{3,16}$

\section{Insulinoterapia}

Aproximadamente entre 50 e $80 \%$ das mulheres com DMG alcançam o controle glicêmico satisfatório com dieta e exercício. No restante, a insulina é a terapia de escolha, já que esta não atravessa a placenta e tem segurança conhecida. $\mathrm{O}$ controle adequado é obtido com uma dose média de 0,5 a $1,0 \mathrm{UI} / \mathrm{kg} /$ dia, porém a dose e o esquema terapêutico inicial devem ser individualizados e posteriormente ajustados conforme os resultados da monitoração da glicemia capilar. Pacientes que apresentem apenas elevação da glicemia pósprandial podem ser adequadamente tratadas com o uso de insulina regular ou ultrarrápida, antes das refeições. Caso tanto a glicemia de jejum quanto as glicemias pós-prandiais estejam elevadas, o esquema de múltiplas doses de insulina, em regime basal bolus, é a melhor opção de tratamento. ${ }^{13}$

Pacientes portadoras de diabetes tipo 2, em uso de hipoglicemiantes orais, devem ser recomendadas a suspender essas medicações e iniciar tratamento com insulina quando estiverem planejando engravidar, uma vez que não há comprovação de segurança do uso de hipoglicemiantes no primeiro trimestre. ${ }^{17}$ Pacientes com diabetes pré-gestacional que já faziam uso prévio de insulina, em geral, necessitam de redução da dose de insulina em 10 a 20\% no primeiro trimestre, devido à maior ocorrência de hipoglicemia nesse período. Após a $18^{\mathrm{a}}$ semana, é frequente a necessidade de aumento progressivo da dose de insulina, sendo que, ao final da gestação, a dose diária de insulina utilizada é, em média, o dobro da dose pré-gestacional. ${ }^{18}$

As insulinas ultrarrápidas lispro e aspart são seguras na gestação e têm a vantagem de pro- 
Tabela 2. Recomendações de ganho de peso na gestação

\begin{tabular}{c|c|c|c}
\hline $\begin{array}{c}\text { Estado nutricional inicial } \\
\text { (IMC) }\end{array}$ & $\begin{array}{c}\text { Ganho total no 1[0] } \\
\text { trimestre (kg) }\end{array}$ & $\begin{array}{c}\text { Ganho semanal no 2[0] e } \\
\text { 3[0] trimestres (kg) }\end{array}$ & $\begin{array}{c}\text { Ganho total na gestação } \\
\text { (kg) }\end{array}$ \\
\hline Baixo peso & 2,3 & 0,5 & $12,5-18,0$ \\
\hline Adequado & 1,6 & 0,4 & $11,5-16,0$ \\
\hline Sobrepeso & 0,9 & 0,4 & $7,0-11,5$ \\
\hline Obesidade & - & 0,2 & $5-9$ \\
\hline
\end{tabular}

porcionar maior flexibilidade de estilo de vida e melhor controle da glicemia pós-prandial do que a insulina regular, embora com a desvantagem do custo mais elevado. ${ }^{11,17,19}$ A insulina ultrarrápida glulisina ainda não tem segurança comprovada na gestação e não deve ser utilizada nessas pacientes.

Recomenda-se que gestantes diabéticas com alto risco de hipoglicemia façam uso da insulina detemir, análogo de insulina de ação longa, cuja segurança na gravidez já foi bem estabelecida. 11,20,21 Estudos com insulina glargina na gestação não demonstraram efeitos adversos, mas apresentam limitações que comprometem a interpretação dos resultados, sendo ainda necessária a realização de estudos prospectivos e controlados para que a segurança desse análogo seja de fato estabelecida.

Pacientes que, antes de engravidar, apresentavam bom controle glicêmico com o uso da terapia de infusão contínua de insulina subcutânea devem manter o tratamento durante a gestação. Por outro lado, não se recomenda o início do uso da bomba de insulina durante a gestação, exceto para pacientes que não obtiveram sucesso terapêutico com os outros esquemas de insulinização, uma vez que há risco de piora do controle glicêmico, cetoacidose e hipoglicemia, no período de início da terapia de infusão contínua. ${ }^{20}$

\section{Hipoglicemiantes orais}

A glibenclamida pode ser utilizada, como uma alternativa à insulina, em mulheres com diabetes mellitus gestacional com mais de 25 semanas de gestação e que apresentem glicemia de jejum menor do que 110 mg/dL. ${ }^{11,21}$

Ao contrário da glibenclamida, a metformina atravessa a barreira placentária. No entanto, estudos recentes demonstram segurança no seu uso em portadoras de diabetes gestacional com mais de 24 semanas e, em seus filhos, até os dois anos de idade. ${ }^{22,23}$ As vantagens são praticidade do uso, ausência de hipoglicemia e baixo custo.

A segurança do uso da glibenclamida e da metformina na gestação em pacientes com diabetes pré-gestacional ainda não foi estabelecida. Não há dados sobre a eficácia e a segurança de outros hipoglicemiantes na gestação.

\section{Acompanhamento}

As mudanças hormonais que ocorrem ao longo da gestação interferem no metabolismo glicídico de forma dinâmica, de modo que o acompanhamento de gestantes diabéticas deve ser feito em consultas frequentes, recomendando-se intervalos quinzenais até a $30^{\mathrm{a}}$ semana e semanais a partir de então. ${ }^{13}$ Como o nível de hemoglobina glicada (HbA1C) reflete o controle glicêmico dos 3 meses anteriores, não é o melhor parâmetro para guiar o tratamento do diabetes na gestação. A monitoração da glicemia capilar é fundamental no seguimento dessas pacientes, recomendando-se aferições diárias antes e 1 hora após o início das três principais refeições, mas podendo ser menos frequente em portadoras de diabetes gestacional em tratamento apenas com dieta.

Pacientes com diabetes prévio têm risco de piora de complicações microvasculares durante a gestação e devem realizar exame oftalmológico, com fundoscopia, antes ou logo após o diagnóstico de gravidez. Caso seja diagnosticada retinopatia, o exame deve ser repetido a cada trimestre e três meses após o parto. Avaliação da função renal e de proteinúria deve ser realizada a cada trimestre. Além da ultrassonografia morfológica de rotina, as portadoras de diabetes pré-gesta- 


\section{Quadro 2.}

Exames em gestantes com diabetes pré-gestacional
Glicemia e hemoglobina glicada - a cada trimestre
Função renal - ureia, creatinina, clearance da creatinina,
proteinúria de 24 horas ou relação proteinúria/creatininúria
em amostra única a cada trimestre
EAS e URC mensais (todas as gestantes com diabetes)
Fundoscopia a cada trimestre
ECG e avaliação cardiológica - $1^{0}$ trimestre
TSH, T4L e anti-TPO

cional devem realizar ecocardiografia fetal entre a $24^{\mathrm{a}}$ e a $28^{\mathrm{a}}$ semana (Quadro 2 ). ${ }^{12}$

O acompanhamento do crescimento fetal e de alterações no líquido amniótico é recomendado para todas as gestantes diabéticas, sendo realizado através de ultrassonografias obstétricas mensais a partir da $26^{\mathrm{a}}$ semana. A identificação de circunferência abdominal acima do percentil 75 ou peso fetal acima do percentil 90 devem levar à intensificação do tratamento. Os exames realizados para avaliação fetal estão listados no quadro 3.

\section{Parto}

Na ausência de complicações, o parto deve ser vaginal e a termo. Nas pacientes em que há dificuldade na obtenção de controle glicêmico, deve-se considerar indução do parto com 38 semanas. Durante o parto, deve-se monitorar a glicemia a cada hora, objetivando-se manter a glicemia entre 72 e $126 \mathrm{mg} / \mathrm{dL}$, podendo ser necessária administração de insulina em infusão contínua. ${ }^{12}$ A cesariana eletiva deve ser considerada apenas na presença de complicações, como peso fetal estimado pela ultrassonografia acima de $4 \mathrm{~kg}$.

\section{Acompanhamento pós-parto}

A insulina deve ser suspensa após o parto em mulheres com DMG e estas devem ser orientadas a realizar um teste oral de tolerância à glicose com 75 g, com 6 a 12 semanas pós-parto para afastar a possibilidade de diabetes.

Pacientes com diabetes pré-gestacional de-
Quadro 3.

\section{Exames para avaliação fetal:}

USG $1^{0}$ trimestre para datação e rastreio de aneuploidia USG morfológica fetal entre 22 e 24 semanas

Ecocardiografia fetal entre 24 e 28 semanas (pacientes com diabetes pré-gestacional)

USG obstétrica nas $26^{\mathrm{a}} / 30^{\mathrm{a}} / 34^{\mathrm{a}} / 36^{\mathrm{a}} / 38^{\mathrm{a}}$ semanas

Dopplervelocimetria mensalmente a partir da $26^{\mathrm{a}}$ semana (apenas em pacientes com diabetes pré-gestacional com vasculopatia ou CIUR)

CTG - bom controle metabólico - semanal a partir da $32^{\mathrm{a}}$ semana

inadequado controle metabólico - 2x/semana a partir da $35^{\mathrm{a}}$ semana

vem ter a dose de insulina diminuída para metade a um terço da dose administrada ao final da gestação ou, opcionalmente, para a dose utilizada antes da gravidez. ${ }^{4}$ Mulheres com diabetes tipo 2 podem fazer uso de metformina ou glibencamida durante a amamentação. ${ }^{12,13}$ Deve-se orientar as pacientes sobre os possíveis métodos contraceptivos e aconselhar sobre a importância de planejamento de futuras gestações.

\section{Referências}

1. Catalano PM, Tyzbir ED, Wolfe RR. Carbohydrate metabolism during pregnancy in control subjects and women with gestational diabetes. Am J Physiol. 1993;264:E60-67.

2. International Association of Diabetes and Pregnancy Study Groups (IADPSG): International Association of Diabetes and Pregnancy Study Groups recommendations of diagnosis and classification of hyperglycemia in pregnancy. Diabetes Care. 2010;33(3):676-82.

3. Metzger BE, Lowe LP, Dyer AR, Trimble ER, Chaovarindr U, Coustan DR, et al. Hyperglycemia and adverse pregnancy outcomes. The HAPO Study Cooperative Research Group. N Engl J Med. 2008;358(19):1991-2002. http://dx.doi.org/10.1056/ NEJMoa0707943

4. Galerneau F, Inzucchi SE. Diabetes Mellitus in pregnancy. Obstet Gynecol Clin North Am. 2004;31:907-33

5. Jovanovic-Peterson L, Peterson CM, Reed GF, Metzger BE, Mills JL, Knopp RH, et al. Maternal postprandial glucose levels and infant birth weith: the Diabetes in Early Pregnancy Study. The National Institute of Child Health and Human Development 
Diabetes in Early Pregnancy Study. Am J Obstet Gynecol. 1991;164:103-11.

6. Ehrenberg HM, Mercer BM, Catalano PM. The interference of obesity and diabetes on the prevalence of macrossomia. Am J Obstet Gynecol. 2004;191(3): 964-8.

7. Nold JL, Georgieff MK. Infants of diabetic mothers. Pediatr Clin North Am. 2004;51:619-37.

8. Petitt DJ, Bennett PH, Knowler WC, Baird HR, Aleck KA. Gestational diabetes mellitus and impaired glucose during pregnancy: long-term effects on obesity and glucose tolerance in the offspring. Diabetes. 1985;34(Suppl 2):119-22.

9. The American College of Obstetrics and Gynecology. Diagnosis and management of pre-eclampsia and eclampsia: ACOG practice bulletin no.33. Obstet Gynecol. 2002;99:159-67.

10. Lauenborg J, Hansen T, Jensen DM, Vestergaard H, Mølsted-Pedersen L, Hornnes P, et al. Increasing incidence of diabetes after gestational diabetes: a long-term follow-up in a danish population. Diabetes Care. 2004;27(5):1194-1199.

11. Blumer I, Hadar E, Hadden DR, Jovanovic L, Mestman JH, Murad MH, et al. Diabetes and pregnancy: an endocrine society clinical practice guideline. J Clin Endocrinol Metab. 2013;98(11):4227-49.

12. Prutsky GJ, Domecq JP, Wang Z, Carranza Leon BG, Elraiyah T, Nabhan M, et al. Glucose targets in pregnant women with diabetes: a systematic review and meta-analysis. J Clin Endocrinol Metab. 2013;98(11):4319-24.

13. Negrato CA, Montenegro Junior RM, Mattar R, Zajdenverg L, Francisco RP, Pereira BG, et al. Dysglycemias in pregnancy: from diagnosis to treatment. Brazilian consensus statement. Diabetol Metab Syndr. 2010;24:2-27.

14. Reader DM. Medical Nutrition Therapy and Lifestyle
Interventions. Diabetes Care. 2007;30:S188-S93.

15. Rasmussen KM, Yaktine AL. IOM (Institute of Medicine) and NRC (National Research Council). Weight Gain During Pregnancy: Reexamining the Guidelines. Washington, DC: The National Academies Press; 2009.

16. Colberg SR, Castorino K, Jovanovič L. Prescribing physical activity for gestational diabetes. World J Diabetes. 2013;4(6):256-62.

17. Negrato CA, Montenegro Junior RM, Von Kostrisch LM, Guedes MF, Mattar R, Gomes MB. Insulin analogues in pregnancy. Arq Bras Endocrinol Metab. 2012;56(7):405-14

18. Oliveira JEP, Milech A. Diabetes mellitus: clínica, diagnóstico, tratamento multidisciplinar. São Paulo: Editora Atheneu; 2004. 362 p.

19. Jovanovic L, Pettitt DJ. Treatment with insulin and its analogs in pregnancies complicated by diabetes. Diabetes Care. 2007;30(S2):S220-4.

20. Mathiesen ER, Hod M, Ivanisevic M, Duran Garcia S, Brøndsted L, Jovanovic L, et al. Detemir in Pregnancy Study Group. Maternal efficacy and safety outcomes in a randomized, controlled trial comparing insulin detemir with NPH insulin in 310 pregnant women with type 1 diabetes. Diabetes Care. 2012;35(10):2012-7.

21. Dhulkotia JS, Ola B, Fraser R, Farrell T. Oral hypoglycemia agents vs. insulin in management of gestational diabetes: a systemic review and metaanalysis. Am J Obstet Gynecol. 2009;203:457.e1-e9.

22. Rowan, JA. A Trial in Progress: Gestational Diabetes. Treatment with metformin compared with insulin (the Metformin in Gestational Diabetes [MiG] trial). Diabetes Care. 2007;30:S214-9.

23. Rowan JA, Rush EC, Obolonkin V, Battin M, Wouldes T, Hague WM. Metformin in gestational diabetes: the offspring follow-up (MiG TOFU): body compositionat 2 years of age. Diabetes Care. 2011;34(10):2279-84. 


\section{Raquel C. Abi-Abib}

Disciplina de Diabetes e Metabologia. Departamento de Medicina Interna. Faculdade de Ciências Médicas. Universidade Estadual do Rio de Janeiro. Rio de Janeiro, RJ, Brasil.

\section{Carolina A. Cabizuca}

Disciplina de Diabetes e Metabologia. Departamento de Medicina Interna. Faculdade de Ciências Médicas. Universidade Estadual do Rio de Janeiro. Rio de Janeiro, RJ, Brasil.

\section{João Regis I. Carneiro}

Disciplina de Diabetes e Metabologia. Departamento de Medicina Interna. Faculdade de Ciências Médicas. Universidade Estadual do Rio de Janeiro. Rio de Janeiro, RJ, Brasil.

\section{Fernanda O. Braga}

Disciplina de Diabetes e Metabologia. Departamento de Medicina Interna. Faculdade de Ciências Médicas. Universidade Estadual do Rio de Janeiro. Rio de Janeiro, RJ, Brasil.

\section{Roberta A. Cobas}

Disciplina de Diabetes e Metabologia. Departamento de Medicina Interna. Faculdade de Ciências Médicas. Universidade Estadual do Rio de Janeiro. Rio de Janeiro, RJ, Brasil.

\section{Marília B. Gomes}

Disciplina de Diabetes e Metabologia. Departamento de Medicina Interna. Faculdade de Ciências Médicas. Universidade Estadual do Rio de Janeiro. Rio de Janeiro, RJ, Brasil.

\section{Guilherme R. de Jesús}

Disciplina de Obstetrícia. Departamento de Obstetrícia. Faculdade de Ciências Médicas. Universidade do Estado do Rio de Janeiro. Rio de Janeiro, RJ, Brasil.

\section{Fátima R. D. Miranda}

Disciplina de Obstetrícia. Departamento Ginecologia e Obstetrícia. Faculdade de Ciências Médicas. Universidade do Estado do Rio de Janeiro. Rio de Janeiro, RJ, Brasil. 